\title{
Everolimus-induced pneumonitis associates with favourable outcome in patients with metastatic renal cell carcinoma
}

\section{Penttila, $\mathrm{P}$.}

\author{
2017-08
}

Penttila , P , Donskov , F , Rautiola , J , Peltola , K , Laukka , M \& Bono , P 2017 , '

Everolimus-induced pneumonitis associates with favourable outcome in patients with

metastatic renal cell carcinoma ' , European Journal of Cancer, vol. 81 , pp. 9-16 . https://doi.org/10.1016/j.ejca.201

http://hdl.handle.net/10138/297790

https://doi.org/10.1016/j.ejca.2017.05.004

publishedVersion

Downloaded from Helda, University of Helsinki institutional repository.

This is an electronic reprint of the original article.

This reprint may differ from the original in pagination and typographic detail.

Please cite the original version. 


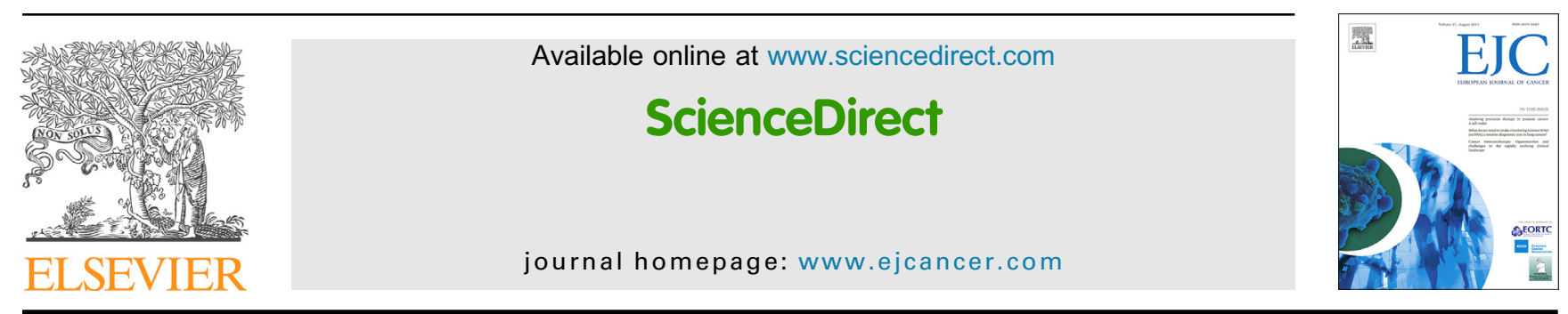

Original Research

\title{
Everolimus-induced pneumonitis associates with favourable outcome in patients with metastatic renal cell carcinoma
}

\author{
P. Penttilä ${ }^{a}{ }^{*}$, F. Donskov ${ }^{b}$, J. Rautiola $^{\text {a }}$, K. Peltola ${ }^{\text {a }}$, M. Laukka ${ }^{\text {a }}$, \\ P. Bono ${ }^{\text {a }}$
}

${ }^{a}$ Comprehensive Cancer Center, Helsinki University Hospital, Helsinki, Finland

${ }^{\mathrm{b}}$ Department of Oncology, Aarhus University Hospital, Aarhus, Denmark

Received 28 February 2017; received in revised form 26 April 2017; accepted 1 May 2017

Available online 3 June 2017

\section{KEYWORDS}

mTOR-inhibitor;

Everolimus;

Pneumonitis
Abstract Background: Mammalian target of rapamycin inhibitors may induce pneumonitis. We analysed the association of pneumonitis with outcomes in everolimus treated metastatic renal cell carcinoma (mRCC) patients.

Patients and methods: Eighty-five $\mathrm{mRCC}$ patients received everolimus at Helsinki University Hospital (cohort A). Computed tomography (CT) verified pneumonitis was correlated with outcome using Kaplan-Meier, Cox regression and logistic regression. An independent cohort of 148 everolimus treated mRCC patients (cohort B) at Aarhus University Hospital was assessed for validation.

Results: In cohort A, CT-verified pneumonitis $(N=29,34.1 \%)$ was associated with improved overall survival (OS) (24.7 versus 8.5 months; $P<0.001$ ), progression-free survival (PFS) (5.5 versus 3.2 months; $P=0.002$ ) and clinical benefit rate (CBR) $57.1 \%$ versus $24.1 \%$ $(P=0.003)$. In multivariate analyses pneumonitis was associated with improved OS (hazard ratio [HR], 0.22; 95\% confidence interval [CI] 0.12-0.44; $P<0.001$ ), PFS (HR 0.37; 95\% CI $0.21-0.66 ; P=0.001$ ) and CBR (odds ratio [OR] 4.11;95\% CI 1.42-11.95; $P=0.01$ ).

In cohort $\mathrm{B}, \mathrm{CT}$-verified pneumonitis $(N=29,19.6 \%)$ was associated with improved OS (12.9 versus 6.0 months; $P=0.02)$, PFS (6.0 versus 2.8 months; $P=0.02)$ and $\mathrm{CBR}$ $(79.3 \%$ versus $39.5 \% ; P<0.001)$. In multivariate analyses pneumonitis was associated with improved OS (HR 0.58; 95\% CI 0.36-0.94; $P=0.03$ ), PFS (HR 0.61; 95\% CI 0.39-0.95; $P=0.03)$ and CBR (OR 5.65; 95\% CI 2.10-15.18; $P=0.001)$.

In a combined multivariate analysis $(N=233)$, with pneumonitis as a time-dependent covariate, CT-verified pneumonitis was associated with longer OS (HR, 0.67; 95\% CI 0.46-0.97;

* Corresponding author: Comprehensive Cancer Center, Helsinki University Hospital, P.O. Box 180, HUS, FIN-00029, Helsinki, University of Helsinki, Finland.

E-mail address: patrick.penttila@helsinki.fi (P. Penttilä). 
$P=0.03)$. Furthermore, in a landmark analysis, pneumonitis was associated with longer OS (17.4 versus 7.8 months; $P=0.01$ ).

Conclusions: Everolimus-induced pneumonitis is associated with improved outcome in patients with mRCC and may serve as a biomarker of everolimus efficacy.

(c) 2017 Elsevier Ltd. All rights reserved.

\section{Introduction}

Everolimus is an orally administrated inhibitor of mammalian target of rapamycin (mTOR), which regulates cell growth, proliferation, survival and angiogenesis [1]. It is a recommended treatment for patients with metastatic renal cell carcinoma (mRCC), whose disease has progressed after initial vascular endothelial growth factor receptor (VEGFR) therapy, either alone [2], or in combination with lenvatinib [3]. Other second-line treatment options are nivolumab [4], cabozantinib [5] and axitinib [6]. Everolimus has also been approved for treatment of progressive neuroendocrine tumours and advanced breast cancer [7-9].

Everolimus has class-specific adverse effects, including rash, pneumonitis, risk of infection, stomatitis, fatigue and metabolic changes. Everolimus induced noninfectious pneumonitis is characterised clinically by difficulty of breathing, often accompanied by a dry, non-productive cough and radiologically by nonspecific, non-infectious and non-malignant infiltrates in the lungs. The reported incidence of pneumonitis during everolimus treatment varies between $13.5 \%$ and $48.7 \%$ [10-12]. The pathogenesis of mTOR-related pneumonitis remains unknown. Suggested mechanisms include a cell-mediated autoimmune response and T-cell-mediated delayed-type hypersensitivity $[13,14]$. In two previous studies involving mRCC patients treated with mTOR-inhibitors, pneumonitis was a marker of stable disease (SD) [15] or prolonged survival [16]. The number of patients treated with everolimus was, however, low ( $N=25$ and $N=100$, respectively) and the studies did not control for potential time-bias from longer treatment.

The objective of this study was to evaluate the predictive and prognostic role of everolimus induced pneumonitis in consecutive patients with $\mathrm{mRCC}$ in two independent patient cohorts.

\section{Materials and methods}

\subsection{Patients and treatment}

A total of 85 consecutive $\mathrm{mRCC}$ patients were treated with everolimus after VEGFR targeted therapy failure at the Cancer Center, Helsinki University Hospital, Finland, between October 18, 2006 and December 31,
2014 (cohort A). Data collected from the hospital case records included patient demographic features, treatments given, adverse events, hospitalisations and outcome data.

A validation series of 148 consecutive patients treated with everolimus between January 25, 2010 and June 6, 2016 at Aarhus University Hospital, Denmark were collected (cohort B).

Everolimus was administered according to standard care until disease progression or unacceptable toxicity.

\subsection{Assessment of tumour response and adverse events}

Response to treatment was assessed by computed tomography at 8-12 week intervals. Treatment efficacy was reported according to Response Evaluation Criteria in Solid Tumours version 1.1 (RECIST) [17]. Adverse events were captured every 4-6 weeks and were graded according to Common Terminology Criteria for Adverse Events (CTCAE) version 3.0 [18].

\subsection{Assessment of pneumonitis}

Medical files, including computed tomography (CT) scan reports, were retrospectively reviewed for pneumonitis and the radiographic studies were subjected to a blinded review by a radiologist for findings indicative of pneumonitis. Patients with confirmed radiologic evidence of pneumonitis (graded per CTCAE version 3.0) were assigned to the CT-verified pneumonitis group (cohort A). To further evaluate the impact of pneumonitis, patients in cohort $\mathrm{B}$ were divided into three groups: patients with pneumonitis verified by $\mathrm{CT}$ (graded per CTCAE version 3.0), patients with clinical pulmonary symptoms, but inconclusive radiological evidence of pneumonitis/pneumonia and patients with no clinical or radiological signs of pneumonitis/ pneumonia.

\subsection{Statistical analysis}

The study population consisted of two independent patient cohorts, cohorts $\mathrm{A}$ and $\mathrm{B}$, and they were analysed separately. The patients' characteristics were described overall and by pneumonitis status. Median follow-up time for patients alive was assessed using Schemper's method. The association of pneumonitis and 
clinical variables were assessed using the Man$\mathrm{n}$-Whitney $\mathrm{U}$ test for continuous data and the Chisquared test for categorical data. The end-points in the study were (i) overall survival (OS) defined as the time from treatment initiation to death, whatever the cause and (ii) progression-free survival (PFS) defined as the time from treatment initiation to the first event (tumour progression or death from any cause) and (iii) clinical benefit rate (CBR) defined as partial response or SD as overall best response per RECIST 1.1. The Kaplan-Meier method was used to estimate the median survival times with $95 \%$ confidence intervals (CIs) for both OS and PFS, censoring the patients who were alive or had no disease progression at the last follow-up visit.

Univariate Cox proportional hazard model was used to assess the association between pneumonitis and OS and PFS in both cohorts separately. The interaction between pneumonitis and cohort was tested in Cox models including cohort, pneumonitis and the interaction term as covariates. Non-significant interaction terms indicated that the association between pneumonitis and OS and PFS was not significantly different in the two cohorts. Multivariate analysis for OS and PFS were performed using Cox proportional hazard models adjusted for age, gender, number of previous treatment lines, Memorial SloanKettering Cancer Center (MSKCC) risk classification for previously treated patients (Karnofsky performance status $<80 \%$, serum haemoglobin $\leq 115 \mathrm{~g} / \mathrm{L}$ for females and $\leq 130 \mathrm{~g} / \mathrm{L}$ for males, corrected calcium $\geq 2.5 \mathrm{mmol} / \mathrm{L}$ ) [19] and pneumonitis. The results are expressed as hazard ratios (HRs) with 95\% CI. The proportional hazards assumption was assessed graphically, obtaining plots of $\log (-\log S(\mathrm{t}))$ versus time and Schoenfeld residuals versus time. Minor violations of the proportional hazards were accepted because the number of patients at risk after 12 months was not adequate to estimate and interpret reliably the plots. Therefore, the models were confirmed using also a shorter follow-up time.

A multivariate logistic regression model was used to investigate the effects of age, gender, MSKCC risk classification and pneumonitis on the CBR. The results are expressed as ORs with $95 \%$ CI.

To control for lead-time bias, a time-dependent Cox regression model, including cohort as a covariate, was conducted. Additionally, a landmark survival analysis with the landmark set at 2.5 months after date of initiation of everolimus treatment was applied. To provide sufficient statistical power, the two patient cohorts were combined $(N=233)$. Per the landmark method, the analysis included patients who had no events before the landmark time point. PFS and OS were defined as the time from the landmark to progression or death from any cause, and pneumonitis status was assessed according to whether pneumonitis had developed up to the landmark time.

All statistical tests were two-sided and Pvalues $<0.05$ were considered as statistically significant.
Analysis was performed using IBM SPSS Statistics for Windows (version 24.0, Armonk, NY, USA, IBM Corp.).

\section{Results}

\subsection{Patient characteristics}

The study population consisted of two patient cohorts: cohort A $(N=85)$ and a validation cohort B $(N=148)$. Patient characteristics for both groups are shown in Table 1. Median follow-up time for patients alive was 44.1 months in cohort A and 37.1 months in cohort B. Median times on everolimus treatment were 2.9 months (range: 0.4-31.2 months) and 2.8 months (range 0.1-46.3 months), respectively. In cohort A, 66 (77.6\%) patients stopped treatment due to progression and 15 $(17.6 \%)$ patients due to adverse events (AE). The remaining four patients $(4.7 \%)$ continued treatment at the time of data cut-off. In cohort B, $85(57.4 \%)$ patients stopped treatment due to progression and $54(36.5 \%)$ patients without progression (33 AE, 21 other). The remaining nine patients $(6.1 \%)$ continued treatment at the time of data cut-off.

OS and PFS for cohort A were 11.0 months $(95 \%$ CI 7.3-14.7 months) and 3.5 months (95\% CI 3.2-3.9 months), respectively, and 6.7 months (95\% CI 5.4-8.1 months) and 2.8 months (95\% CI 2.5-3.2 months), respectively, for cohort $\mathrm{B}$.

Of the patients in cohort $\mathrm{A}$ and $\mathrm{B}, 11.8 \%$ and $19.6 \%$, respectively, received everolimus after three or more treatment lines.

\subsection{Pneumonitis and outcome}

In cohort A, 29 (34.1\%) patients had CT-verified pneumonitis during everolimus treatment. Eight cases were grade I (27.6\%), 18 cases grade II $(62.1 \%)$ and three cases were grade III $(10.3 \%)$. No grade IV (life-threatening) pneumonitis was recorded. In cohort B, 29 (19.6\%) patients had CT-verified pneumonitis. Among patients with pneumonitis, median time to onset was 2.4 months (range 0.4-7.5 months) in cohort A and 2.8 months (range 0.1-14.1 months) in cohort B.

In univariate analysis, patients with pneumonitis had longer OS (cohort A: 24.7 versus 8.5 months; $P<0.001$; cohort B: 12.9 versus 6.0 months; $P=0.02$ ) and PFS (cohort A: 5.5 versus 3.2 months; $P=0.002$; cohort B: 6.0 versus 2.8 months; $P=0.02$ ) as compared to patients with no pneumonitis. Kaplan-Meier curves for $\mathrm{OS}$ and PFS are depicted in Fig. 1A-D. As the interactions between cohort and pneumonitis were nonsignificant ( $\mathrm{P}=0.36$ for $\mathrm{OS}$ and $\mathrm{P}=0.69$ for $\mathrm{PFS})$, the two patient cohorts were combined. In the combined data using Cox regression model with cohort as a covariate, pneumonitis was significantly associated with 
Table 1

Characteristics of patients with and without pneumonitis in cohorts' A and B.

\begin{tabular}{|c|c|c|c|c|c|c|c|c|c|}
\hline & & \multicolumn{3}{|c|}{ Cohort A $(N=85)$} & \multirow[t]{3}{*}{$P$} & \multicolumn{3}{|c|}{ Cohort B $(N=148)$} & \multirow[t]{3}{*}{$P$} \\
\hline & & \multicolumn{2}{|l|}{ Pneumonitis } & \multirow{2}{*}{$\frac{\text { Total }}{N=85}$} & & \multicolumn{2}{|l|}{ Pneumonitis } & \multirow{2}{*}{$\frac{\text { Total }}{N=148}$} & \\
\hline & & $\begin{array}{l}\text { No }(N=56) \\
(65.9 \%)\end{array}$ & $\begin{array}{l}\text { Yes }(N=29) \\
(34.1 \%)\end{array}$ & & & $\begin{array}{l}\text { No }(N=119) \\
(80.4 \%)\end{array}$ & $\begin{array}{l}\text { Yes }(N=29) \\
(19.6 \%)\end{array}$ & & \\
\hline \multirow[t]{2}{*}{ Gender } & Male & $33(58.9)$ & $19(65.5)$ & $52(61.2)$ & 0.55 & 84 (70.6) & $20(69.0)$ & $104(70.3)$ & 0.86 \\
\hline & Female & $23(41.1)$ & $10(34.5)$ & $33(38.8)$ & & $35(29.4)$ & $9(31.0)$ & $44(29.7)$ & \\
\hline \multirow[t]{2}{*}{ Age } & Median & 66.5 & 63.0 & 66.0 & 0.71 & 65.0 & 63.0 & 64.0 & 0.27 \\
\hline & Range & $24-87$ & $48-82$ & $24-87$ & & $39-81$ & $40-77$ & $39-81$ & \\
\hline \multirow[t]{2}{*}{ Karnofsky $<80 \%$} & Yes & $18(32.1)$ & $7(24.1)$ & $21(24.7)$ & 0.44 & $44(37.0)$ & $6(20.7)$ & $50(33.8)$ & 0.10 \\
\hline & No & $38(67.9)$ & $22(75.9)$ & $64(75.3)$ & & $75(63.0)$ & $23(79.3)$ & $98(66.2)$ & \\
\hline \multirow[t]{2}{*}{ Prior nephrectomy ${ }^{\mathrm{a}}$} & Yes & 44 (78.6) & $26(89.7)$ & $70(82.4)$ & 0.20 & $89(74.8)$ & $27(93.1)$ & $116(78.4)$ & 0.03 \\
\hline & No & $12(21.4)$ & $3(10.3)$ & $15(17.6)$ & & $30(25.2)$ & $2(6.9)$ & 32 (21.6) & \\
\hline \multirow[t]{3}{*}{ Histology } & Clear cell & $37(80.4)$ & $25(96.2)$ & $62(86.1)$ & 0.06 & $107(93.0)$ & $26(96.3)$ & $133(93.7)$ & 0.53 \\
\hline & Non-clear cell & $9(19.6)$ & $1(3.8)$ & $10(13.9)$ & & $8(7.0)$ & $1(3.7)$ & $9(6.3)$ & \\
\hline & Missing & 10 & 3 & 13 & & 4 & 2 & 6 & \\
\hline \multirow[t]{3}{*}{ MSKCC classification } & Favourable & $14(25.9)$ & $12(42.9)$ & $26(31.7)$ & 0.12 & $26(21.8)$ & $8(27.6)$ & $34(23.0)$ & 0.68 \\
\hline & Intermediate & $19(35.2)$ & $11(39.3)$ & $30(36.6)$ & & $51(42.9)$ & $13(44.8)$ & $64(43.2)$ & \\
\hline & Poor & $21(38.9)$ & $5(17.9)$ & $26(31.7)$ & & $42(35.3)$ & $8(27.6)$ & $50(29.6)$ & \\
\hline \multirow{3}{*}{$\begin{array}{l}\text { No. of previous } \\
\text { treatment lines }\end{array}$} & 1 & $27(48.2)$ & $11(37.9)$ & $38(44.7)$ & 0.18 & $62(52.1)$ & $11(37.9)$ & 73 (49.3) & 0.32 \\
\hline & 2 & $25(44.6)$ & $12(41.4)$ & $37(43.5)$ & & $36(31.1)$ & $10(34.5)$ & $46(31.1)$ & \\
\hline & $\geq 3$ & $4(7.1)$ & $6(20.7)$ & $10(11.8)$ & & $21(17.6)$ & $8(27.6)$ & $29(19.6)$ & \\
\hline
\end{tabular}

MSKCC, Memorial Sloan-Kettering Cancer Center.

${ }^{\mathrm{a}}$ In cohort B, patients with pneumonitis had prior nephrectomy more often than patients without pneumonitis $(\mathrm{P}=0.03)$.
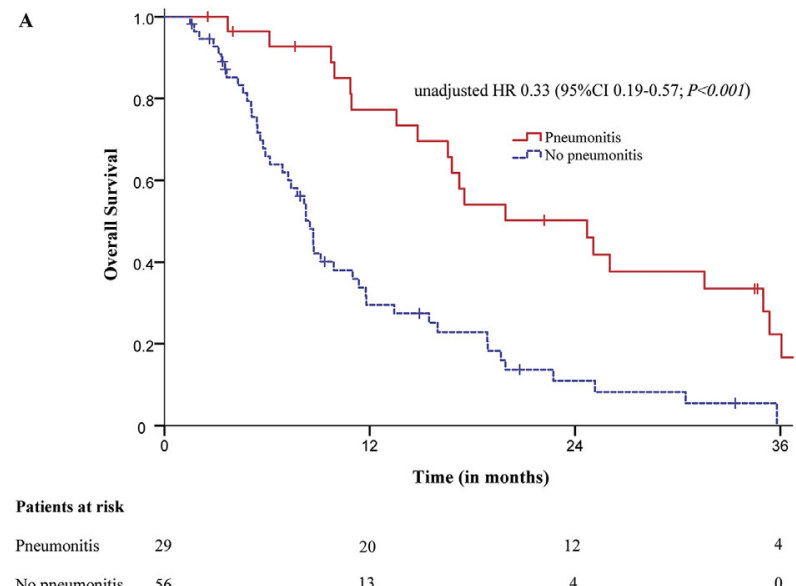

C

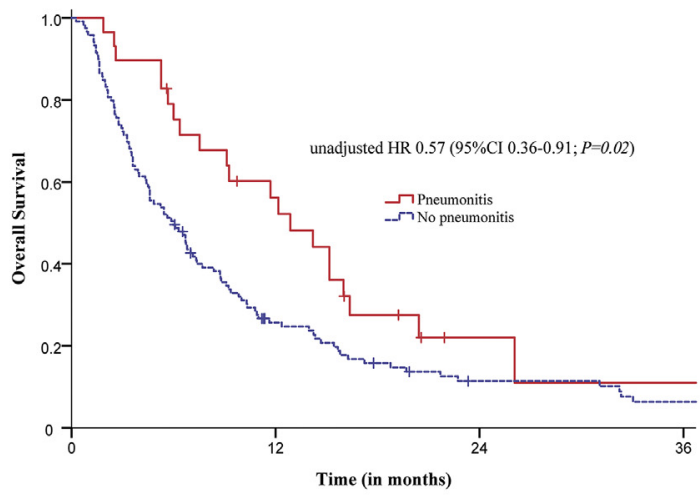

Patients at risk

Pneumonitis 29

No pneumonitis 119
14
B

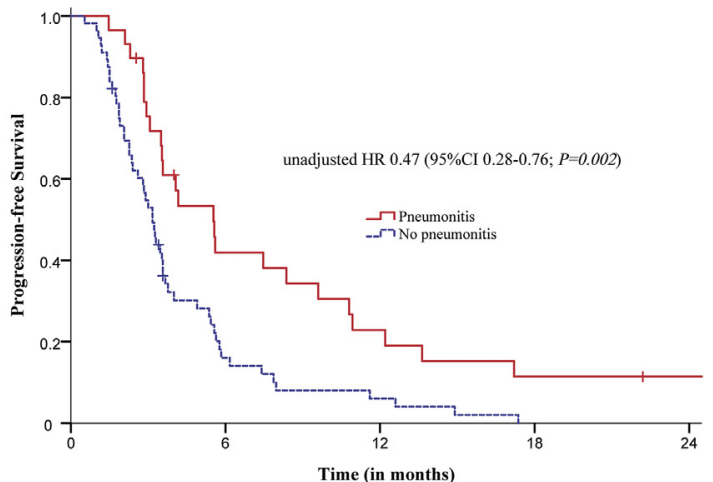

Patients at risk

Pneumonitis 29

No pneumonitis 56

D

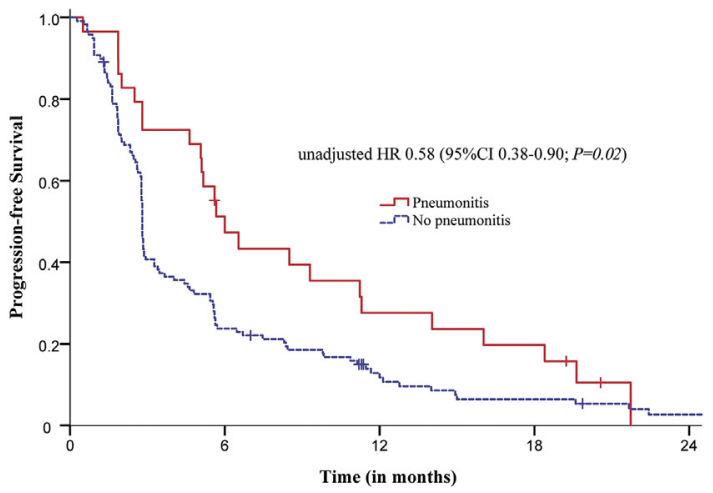

Patients at risk

Pneumonitis 29

No pneumonitis 119
13

Fig. 1. Kaplan-Meier survival curves for patients with pneumonitis, (A) overall survival and (B) progression-free survival in cohort A; (C) overall survival and (D) progression-free survival in cohort B. 
OS (HR 0.49, 95\% CI 0.34-0.69, $\mathrm{P}<0.001$ ) and with PFS (HR 0.53, 0.38-0.73, P $<0.001$ ). Other potential factors associated with OS and PFS are shown in Supplementary Tables $1 \mathrm{~A}$ and B.

The CBR was $57.1 \%(16 / 28)$ and $79.3 \%(23 / 29)$ in patients with pneumonitis as compared to $24.1 \%(13 / 54)$ and $39.5 \%(47 / 119)$ in patients with no pneumonitis, in cohorts A and $\mathrm{B}$, respectively $(P=0.004)(P<0.001)$.

\subsection{Multivariate analyses}

To investigate the independent association of pneumonitis on outcome, we performed a multivariate survival analysis adjusted for age, gender, number of previous treatment lines and MSKCC risk classification for previously treated patients. In the analyses pneumonitis was significantly associated with both longer OS (cohort A: HR, 0.22; 95\% CI 0.12-0.44; $P<0.001$; cohort B: HR $0.58 ; 95 \%$ CI $0.36-0.94 ; P=0.03$ ) and PFS (cohort A: HR $0.37 ; 95 \%$ CI $0.21-0.66 ; P=0.001$; cohort B: HR $0.61 ; 95 \%$ CI $0.39-0.95 ; P=0.03$ ).

A multivariate logistic regression analysis was performed in both groups to ascertain the effects of age, gender, MSKCC risk classification and pneumonitis on the CBR. In cohort A, patients with pneumonitis were 4.11 times more likely to have clinical benefit to everolimus as compared to patients with no pneumonitis during treatment (odds ratio [OR] 4.11; 95\% CI $1.42-11.95 ; P=0.01)$. In cohort $B$ patients with pneumonitis were 5.65 times more likely to have clinical benefit as best response as compared to patients with no pneumonitis during treatment (OR 5.65; 95\% CI 2.10-15.18; $P=0.001)$.

To control for potential lead-time bias, we performed a multivariate survival analysis with pneumonitis as a time-dependent covariate. For this purpose, we combined the two patient cohorts into one $(N=233)$. In a Cox regression model adjusted for age, gender, cohort, number or previous treatment lines and MSKCC risk classification for previously treated patients, CT-verified pneumonitis was independently associated with longer OS (HR, 0.67; 95\% CI 0.46-0.97; $P=0.03$ ).

\subsection{Landmark analysis}

After excluding patients who progressed or died before the landmark time point, 160 patients were included in the landmark analysis. In the analysis, CT-verified pneumonitis $(N=30,18.8 \%)$ was associated with longer OS (17.4 months [95\% CI 5.4-29.5] versus 7.8 months [95\% CI 6.1-9.5]; $P=0.01$ ).

\subsection{Subgroup analyses}

To further elucidate the nature of pneumonitis we divided patients in cohort B into three groups: Patients with pneumonitis verified by CT $(N=29)$, patients with clinical symptoms of pneumonitis/pneumonia $(N=25)$ and patients with no clinical or radiological signs of pneumonitis/pneumonia $(N=94)$. These groups were evenly balanced regarding baseline clinical factors, but patients with CT-verified pneumonitis and patients with clinical symptoms of pneumonitis/pneumonia were more likely to have prior nephrectomy $(93.1 \%$ and $88.0 \%$; respectively) versus no pneumonia/pneumonitis $(71.3 \%$; $P<0.001$ ).

Results regarding OS, PFS and CBR for the three groups are shown in Table 2. Interestingly, there was no statistically significant difference in OS $(P=0.24)$, PFS $(P=0.96)$ or CBR $(P=0.37)$ between patients with $\mathrm{CT}$-verified pneumonitis and patients with clinical symptoms of pneumonitis/pneumonia.

\section{Discussion}

To the best of our knowledge, this is the first study to establish a favourable association between pneumonitis and everolimus treatment in all efficacy end-points (PFS, OS and CBR) and to validate the findings in an independent patient cohort. In patients with CT-verified pneumonitis, OS and PFS were significantly longer. Patients without CT-verified pneumonitis were more than four times more likely to have progressive disease as their best response as compared to patients with pneumonitis. Pneumonitis remained significantly

Table 2

Overall survival, progression-free survival and clinical benefit rate for patients with verified pneumonitis, pneumonitis/pneumonia and patients with no pneumonitis/pneumonia (cohort B).

\begin{tabular}{|c|c|c|c|c|c|c|c|c|c|}
\hline & \multirow[t]{2}{*}{$N$} & \multicolumn{2}{|l|}{ Overall survival } & \multirow[t]{2}{*}{$P$} & \multicolumn{2}{|c|}{ Progression-free survival } & \multirow[t]{2}{*}{$P$} & \multirow{2}{*}{$\begin{array}{l}\text { Clinical } \\
\text { benefit rate } \\
\mathrm{PR}+\mathrm{SD} \%\end{array}$} & \multirow[t]{2}{*}{$P$} \\
\hline & & Median $(95 \% \mathrm{CI})$ & $\operatorname{HR}(95 \% \mathrm{CI})$ & & Median $(95 \%$ CI $)$ & HR $(95 \% \mathrm{CI})$ & & & \\
\hline $\begin{array}{l}\text { No pneumonitis/ } \\
\text { pneumonia }\end{array}$ & 94 & $5.2(3.5-6.9)$ & 1.00 & Reference & $2.8(2.7-2.8)$ & 1.00 & Ref. & 31.9 & Ref. \\
\hline $\begin{array}{l}\text { Pneumonitis/ } \\
\text { Pneumonia }\end{array}$ & 25 & $8.7(4.8-12.7)$ & $0.78(0.49-1.25)$ & 0.30 & $5.6(3.5-7.8)$ & $0.48(0.30-0.78)$ & 0.003 & 68.0 & 0.002 \\
\hline $\begin{array}{l}\mathrm{CT} \text { verified } \\
\text { pneumonitis }\end{array}$ & 29 & $12.9(8.9-16.8)$ & $0.54(0.33-0.87)$ & $0.01\left({ }^{\mathrm{a}} 0.24\right)$ & $6.0(4.5-7.5)$ & $0.47(0.30-0.74)$ & $\begin{array}{l}\mathbf{0 . 0 0 1} \\
\left({ }^{\mathrm{a}} 0.96\right)\end{array}$ & 79.3 & $\begin{array}{l}<0.001 \\
\left({ }^{\mathrm{a}} 0.37\right)\end{array}$ \\
\hline
\end{tabular}

CI, confidence interval; HR, hazard ratio; CT, computed tomography; PR, partial response; SD, stable disease.

${ }^{\text {a }} \mathrm{P}$-values comparing CT-verified pneumonitis and pneumonitis/pneumonia. 
associated with favourable outcome when adjusted for baseline clinical factors. The implication is that CTverified pneumonitis may serve as a biomarker of treatment efficacy in $\mathrm{mRCC}$ patients treated with everolimus.

Previous research in $\mathrm{mRCC}$ patients treated with VEGF targeted therapies has demonstrated that certain mechanism-based treatment-related adverse events, i.e. hypertension, neutropenia and thrombocytopaenia, reflect on-target effects and correlate with clinical efficacy [20-23]. The incorporation of these biomarkers into clinical practice may provide support in clinical decision-making, when considering whether a given treatment should be continued. Thus, pneumonitis may serve as a biomarker during everolimus therapy. For mTOR-inhibitors, such surrogate markers of treatment efficacy have not previously been defined. The pathogenesis of mTOR-inhibitor-related pneumonitis, however, remains unknown. A study investigating sirolimusassociated pneumonitis displayed lymphocytic alveolitis with increased amounts of CD4-positive lymphocytes in bronco alveolar lavage fluid, suggesting a cell-mediated autoimmune response [13]. Similar findings have been published regarding methotrexate-induced pneumonitis [24]. Other pathogenic mechanisms include T-cell mediated delayed-type hypersensitivity and dose-related effects $[13,14]$. Precise underlying mechanisms, whereby mTOR-inhibitor treatment leads to pneumonitis, and especially how this leads to clinical benefit, are yet to be elucidated and require further investigation.

Atkinson et al. previously reported that pneumonitis was associated with longer OS in patients with $\mathrm{mRCC}$ treated with everolimus/temsirolimus [16]. However, PFS and response rates were not reported. Dabydeen et al. demonstrated that mean tumour shrinkage and SD by RECIST were significantly higher in patients with pneumonitis, but did not report PFS or OS results [15]. Our data are in line with these preliminary results and confirm the intriguing finding of improved outcome in patients who develop pneumonitis. Despite early onset of pneumonitis (median 2.4 and 2.8 months), we controlled for potential time bias as patients that benefit from treatment will be treated for a longer period and therefore have a higher chance of developing pneumonitis; the Cox regression analysis using pneumonitis as a time-dependent covariate showed pneumonitis as an independent predictor of improved OS. Furthermore, a landmark analysis, controlling for early deaths and thereby lower chance of developing pneumonitis, demonstrated that pneumonitis was significantly associated with longer OS. The landmark method has some limitations, most importantly, loss of power due to omission of early events and dependence on the choice of the landmark time. However, given that both methods showed similar results for OS consistent with the primary analyses, it is unlikely that our results were impacted by time bias.
The incidence of CT-verified pneumonitis was $34.1 \%$ and $19.6 \%$ in cohorts A and B, respectively. Previously reported incidence of pneumonitis in patients with $\mathrm{mRCC}$ varies between $13.5 \%$ and $48.7 \%[10,11,25]$. This wide variation in reported incidence might be due to the often non-specific radiological appearance and clinical symptoms of mTOR-inhibitor-related pneumonitis resulting in underreporting this adverse event. In addition, many of the studies have relatively few patients. A meta-analysis $(N=2233)$ of published randomised prospective trials in breast cancer, neuroendocrine tumours and mRCC reported an all grade pulmonary toxicity of only $10.4 \%$ among patients treated with mTOR-inhibitors [26]. In our smaller study, we observed a higher incidence of pneumonitis in two independent consecutive patient cohorts using a rigorous approach, emphasising the importance of evaluating the implementation of new therapies in a real-world setting. Nonetheless, we recognise this as a limitation in our study, since differentiating pneumonitis from other incidental interstitial lung diseases as well as from early signs of infection (e.g. viral pneumonia) can be difficult. However, when comparing the results of the subgroup analysis performed in cohort $\mathrm{B}$, there was no significant difference in outcome between patients with CT-verified pneumonitis and patients with clinical symptoms of pneumonitis/pneumonia, and both subgroups had longer PFS and higher CBR than the patients without evidence of pneumonitis/pneumonia. In consideration of the difficulty in differentiating pneumonitis from other diseases of the lung parenchyma, we find the implications of these subgroup analyses clinically relevant and in support of our notion.

Other potential limitations of our study were that the patient cohorts represent a heterogeneous group of patients including different histological variants of RCC. Additionally, data were lacking regarding different radiologic patterns of pneumonitis, concomitant medication and comorbidities, including pre-existing pulmonary dysfunction possibly predisposing patients to pneumonitis. Although retrospective in nature, our study was based on consecutive patients in two independent patient cohorts.

Previous research on transplant recipients receiving mTOR-inhibitors, has shown that pneumonitis is relatively unaggressive and reversible on drug discontinuation, although more severe lung toxicity may infrequently occur [14,27]. The RECORD-1 (REnal Cell cancer treatment with Oral RAD001 given Daily) trial demonstrated that most cases of pneumonitis were manageable and may be resolved by dose reduction or treatment withdrawal, or corticosteroid administration [10]. Recent reviews addressing the issues regarding management of mTOR-inhibitor-associated pneumonitis, suggested that everolimus treatment can be continued, or temporarily interrupted, among patients with mild symptoms (grades I to II). Corticosteroids, 
and occasionally antibiotics, may be used for troublesome symptoms if dose alteration proves ineffective. Even in more severe cases of pneumonitis (grade III), reintroduction of everolimus after treatment interruption is a viable option $[28,29]$. Our clinical practice and experience is in line with these recommendations.

We conclude that everolimus-induced pneumonitis is associated with improved outcome in patients with mRCC and may serve as a biomarker of everolimus efficacy.

\section{Funding}

This research did not receive any specific grant from funding agencies in the public, commercial or not-forprofit sectors.

\section{Conflict of interest statement}

Patrick Penttilä has received honoraria from Novartis. Frede Donskov has received research funding from Pfizer, GSK and Novartis. Katriina Peltola has received honoraria from Novartis, BMS, Pfizer, Amgen, Astellas, Sanofi, Merck and Eli Lilly. Petri Bono has received research funding from Novartis and honoraria from Pfizer, Novartis, Orion Pharma, BMS and MSD. The other remaining authors have declared no conflicts of interest.

\section{Appendix A. Supplementary data}

Supplementary data related to this article can be found at http://dx.doi.org/10.1016/j.ejca.2017.05.004.

\section{References}

[1] Brugarolas J. Renal-cell carcinoma-molecular pathways and therapies. N Engl J Med 2007;356(2):185-7.

[2] Escudier B, Porta C, Schmidinger M, Rioux-Leclercq N, Bex A, Khoo V, et al. Renal cell carcinoma: ESMO Clinical Practice Guidelines for diagnosis, treatment and follow-up. Ann Oncol 2016;27(suppl 5):v58-68.

[3] Motzer RJ, Hutson TE, Glen H, Michaelson MD, Molina A, Eisen T, et al. Lenvatinib, everolimus, and the combination in patients with metastatic renal cell carcinoma: a randomised, phase 2, open-label, multicentre trial. Lancet Oncol 2015;16(15): 1473-82.

[4] Motzer RJ, Escudier B, McDermott DF, George S, Hammers HJ, Srinivas $\mathrm{S}$, et al. Nivolumab versus everolimus in advanced renalcell carcinoma. N. Engl J Med 2015;373(19):1803-13.

[5] Choueiri TK. Cabozantinib versus everolimus in advanced renal cell carcinoma (METEOR): final results from a randomised, open-label, phase 3 trial. Lancet Oncol 2016;17(7):917.

[6] Rini BI, Escudier B, Tomczak P, Kaprin A, Szczylik C, Hutson TE, et al. Comparative effectiveness of axitinib versus sorafenib in advanced renal cell carcinoma (AXIS): a randomised phase 3 trial. Lancet 2011;378(9807):1931-9.

[7] Yao JC, Shah MH, Ito T, Bohas CL, Wolin EM, Van Cutsem E, et al. Everolimus for advanced pancreatic neuroendocrine tumors. N Engl J Med 2011;364(6):514-23.
[8] Yao JC, Fazio N, Singh S, Buzzoni R, Carnaghi C, Wolin E, et al. Everolimus for the treatment of advanced, non-functional neuroendocrine tumours of the lung or gastrointestinal tract (RADIANT-4): a randomised, placebo-controlled, phase 3 study. Lancet 2016;387(10022):968-77.

[9] Baselga J, Campone M, Piccart M, Burris 3rd HA, Rugo HS, Sahmoud T, et al. Everolimus in postmenopausal hormonereceptor-positive advanced breast cancer. N Engl J Med 2012; 366(6):520-9.

[10] White DA, Camus P, Endo M, Escudier B, Calvo E, Akaza H, et al. Noninfectious pneumonitis after everolimus therapy for advanced renal cell carcinoma. Am J Respir Crit Care Med 2010; 182(3):396-403.

[11] Amato RJ, Jac J, Giessinger S, Saxena S, Willis JP. A phase 2 study with a daily regimen of the oral mTOR inhibitor RAD001 (everolimus) in patients with metastatic clear cell renal cell cancer. Cancer 2009;115(11):2438-46.

[12] Soria JC, Shepherd FA, Douillard JY, Wolf J, Giaccone G, Crino L, et al. Efficacy of everolimus (RAD001) in patients with advanced NSCLC previously treated with chemotherapy alone or with chemotherapy and EGFR inhibitors. Ann Oncol 2009; 20(10):1674-81.

[13] Morelon E, Stern M, Israel-Biet D, Correas JM, Danel C, Mamzer-Bruneel MF, et al. Characteristics of sirolimusassociated interstitial pneumonitis in renal transplant patients. Transplantation 2001;72(5):787-90.

[14] Pham P-TT. Sirolimus-associated pulmonary toxicity. Transplantation 2004;77(8):1215.

[15] Dabydeen DA, Jagannathan JP, Ramaiya N, Krajewski K, Schutz FA, Cho DC, et al. Pneumonitis associated with mTOR inhibitors therapy in patients with metastatic renal cell carcinoma: incidence, radiographic findings and correlation with clinical outcome. Eur J Cancer 2012;48(10):1519-24.

[16] Atkinson BJ, Cauley DH, Ng C, Millikan RE, Xiao L, Corn P, et al. Mammalian target of rapamycin (mTOR) inhibitorassociated non-infectious pneumonitis in patients with renal cell cancer: predictors, management, and outcomes. BJU Int 2014; 113(3):376-82.

[17] Eisenhauer EA, Therasse P, Bogaerts J, Schwartz LH, Sargent D, Ford R, et al. New response evaluation criteria in solid tumours: revised RECIST guideline (version 1.1). Eur J Cancer 2009;45(2): $228-47$.

[18] Trotti A, Colevas A, Setser A, Rusch V, Jaques D, Budach V, et al. CTCAE v3.0: development of a comprehensive grading system for the adverse effects of cancer treatment. Semin Radiat Oncol 2003;13(3):176-81.

[19] Motzer RJ, Bacik J, Schwartz LH, Reuter V, Russo P, Marion S, et al. Prognostic factors for survival in previously treated patients with metastatic renal cell carcinoma. J Clin Oncol 2004;22(3): 454-63.

[20] Rodriguez-Vida A, Strijbos M, Hutson T. Predictive and prognostic biomarkers of targeted agents and modern immunotherapy in renal cell carcinoma. ESMO Open 2016;1(3).

[21] Donskov F, Heng DY, Rini BI. On-treatment biomarkers in metastatic renal cell carcinoma: towards individualization of prognosis? Expert Rev Anticancer Ther 2017;17(2):97-9.

[22] Ravaud A. Clinical biomarkers of response in advanced renal cell carcinoma. Ann Oncol 2013;24(12):2935.

[23] Soerensen AV, Geertsen PF, Christensen IJ, Hermann GG, Jensen NV, Fode K, et al. A five-factor biomarker profile obtained week 4-12 of treatment for improved prognostication in metastatic renal cell carcinoma: results from DARENCA study 2. Acta Oncol 2016;55(3):341-8.

[24] Schnabel A, Richter C, Bauerfeind S, Gross WL. Bronchoalveolar lavage cell profile in methotrexate induced pneumonitis. Thorax 1997;52(4):377-9.

[25] Motzer RJ, Escudier B, Oudard S, Hutson TE, Porta C, Bracarda S. Phase 3 trial of everolimus for metastatic renal cell 
carcinoma: final results and analysis of prognostic factors. Cancer 2010;116

[26] Iacovelli R, Palazzo A, Mezi S, Morano F, Naso G, Cortesi E. Incidence and risk of pulmonary toxicity in patients treated with mTOR inhibitors for malignancy. A meta-analysis of published trials. Acta Oncol 2012;51(7):873-9.

[27] Kahan BD, Knight R, Schoenberg L, Pobielski J, Kerman RH, Mahalati K, et al. Ten years of sirolimus therapy for human renal transplantation: the University of Texas at Houston experience. Transpl Proc 2003;35(3 Suppl):25s-34s.
[28] Willemsen AE, Grutters JC, Gerritsen WR, van Erp NP, van Herpen CM, Tol J. mTOR inhibitor-induced interstitial lung disease in cancer patients: comprehensive review and a practical management algorithm. Int J Cancer 2016;138(10):2312-21.

[29] Porta C, Osanto S, Ravaud A, Climent MA, Vaishampayan U, White DA, et al. Management of adverse events associated with the use of everolimus in patients with advanced renal cell carcinoma. Eur J Cancer 2011;47(9):1287-98. 